\title{
THE DYNAMICS OF HEMERYTHRIN AND HEMERYTHRIN DERIVATIVES
}

\author{
FRANCISCO CARRASCOZA ${ }^{a, b, c}$, ADRIAN M.V. BRÂNZANIC ${ }^{a, d}$, \\ RADU SILAGHI-DUMITRESCU
}

\begin{abstract}
The non-heme diiron protein hemerythrin $(\mathrm{Hr})$ has been considered as a possible alternative for semi-artificial oxygen carriers ("blood substitutes"). Studies on the stability of its octameric structure have been attempted by attaching spin labels to analyze its electron paramagnetic spectroscopy (ESR) signals. Reported here are molecular dynamics results of $\mathrm{Hr}$ bound with 1-oxyl-2,2,5,5-tetramethylpyrroline-3methyl (MTSSL) at the Cys51 position. Our results show that the Hr-MTSSL complex is less stable than its native form. These findings help to explain ESR signals obtained experimentally. Also, these results are crucial to understand the limitations of PEGylated spin labels for protein structural analysis and suggest the need or further exploration of other alternatives.
\end{abstract}

Keywords: hemerythrin, molecular dynamics, spin label, blood substitute, diiron

\section{INTRODUCTION}

Hemerythrin $(\mathrm{Hr})$ is a homo-octameric alpha-helical protein with a non-heme diiron active site found in marine worms or bacteria, and has been studied as a potential alternative to hemoglobin as starting material for semiartificial oxygen carriers (SARTOC, or "blood substitutes") either in PEGylated form or in glutaraldehyde-polymerized form.[1-8] The stability of these materials under physiologically relevant conditions has received little attention so

a Babeş-Bolyai University, Faculty of Chemistry and Chemical Engineering, 11 Arany Janos str., RO-400028, Cluj-Napoca, Romania

b Institute of Computer Science, Poznan University of Technology, UI. Piotrowo 2, 61-138, Poznan, Poland

c European Centre for Bioinformatics and Genetics ECBiG, Ul. Piotrowo 2, 61-138, Poznan, Poland

d Babeş-Bolyai University, Institute of Interdisciplinary Research in Bio-Nano-Sciences, 42 Treboniu Laurian str., RO- 400271, Cluj-Napoca, Romania

Corresponding author: radu.silaghi@ubbcluj.ro 
far. Recently, (1-oxyl-2,2,5,5-tetramethylpyrroline-3methyl)methanethiosulfonate (MTSSL) has been attached at the Cys51 position of each monomer of $\mathrm{Hr}$ in order to analyze EPR signals that can render information about the stability of the $\mathrm{Hr}$ octameric structure.[9,10]

Earlier, all-atom molecular dynamics on $\mathrm{Hr}$ has been performed to explore dioxygen permeation to its diiron active site - but mostly as a reference to methane monooxygenase pathways.[11] On the other hand, more relevant for SARTOC would be solvent accessibility (hence the polarity) of the oxy-diiron site, since this would control the autooxidation rate which is in turn responsible for a good part of the side effect of SARTOC candidates. [8,12-15]

Reported here is a theoretical attempt to explore the behavior of MTSSL-labeled Hr. Using molecular dynamics we try to understand the experimental results, and to describe whenever MTSSL label can be a suitable compound to analyze $\mathrm{Hr}$ in vitro. Our results show that when the octameric $\mathrm{Hr}$ is fully PEGylated, there is a pattern of decreased stability, probably due to the inter monomer clashes induced by the spin label. This can be a suitable explanation for the observed EPR signals.[9] These findings may be of interest also in understanding how certain cross-linkers used in blood substitutes affect $\mathrm{Hr}$ and hemoglobin derivatives in its relationship structure and behavior.

\section{RESULTS AND DISCUSSION}

One goal of MD simulations with $\mathrm{Hr}$ is to establish the degree of mobility within the monomer, and the manner in which chemical derivatization with PEG or with MTSSL (spin label) would affect the results. Measurements of distances and volumes intra and inter monomer were thus taken in order to observe the effect of the label across the time. Thus, Figure 1 reveals that the spin-labeled cysteine residue lies sufficiently close to the inter-monomer interface in order to disrupt monomer-monomer interactions. Indeed, the distance between the a carbons of labeled Cys51 (Cyx51) in one chain and Asp23 in the neighboring chain, is on average $5 \AA$ longer in the spinlabelled structure - an increase sufficient to disrupt most of the typical intermolecular forces (hydrogen bonds and hydrophobic interactions especially). This difference is already seen in the geometry-optimized structures, and is maintained throughout the 5 nanoseconds of MD simulation - albeit with a slight decrease in time. 


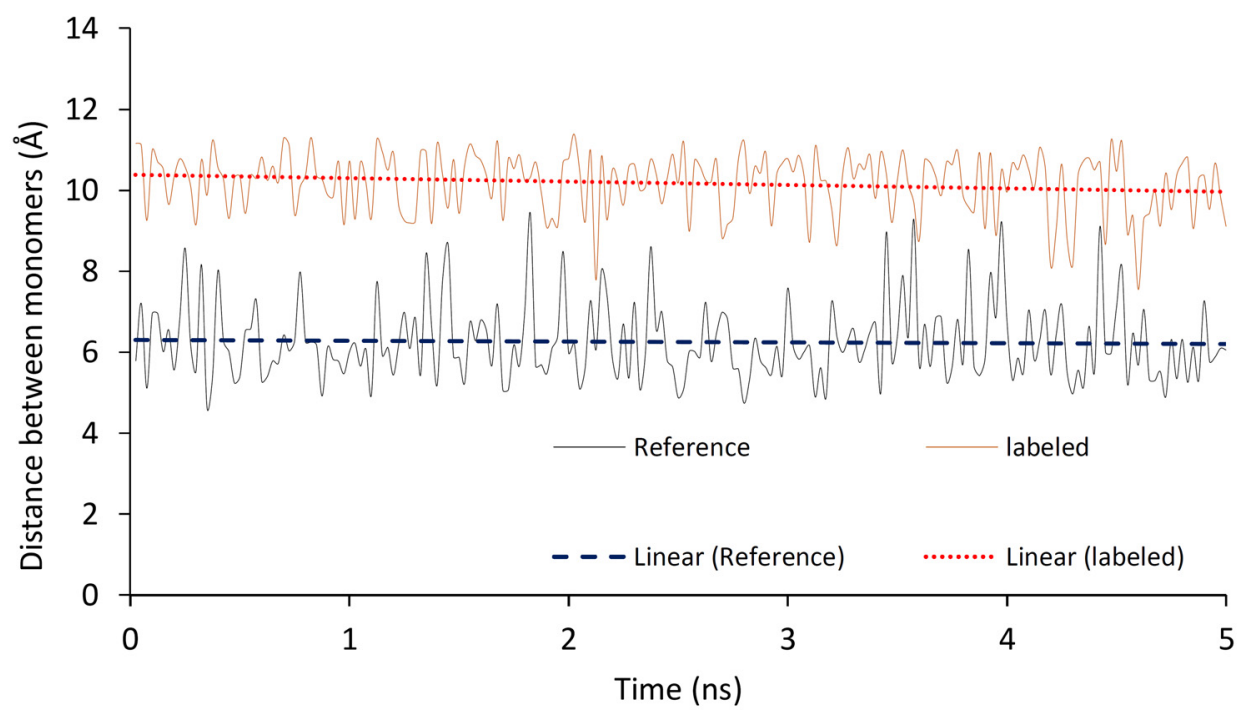

Figure 1. Distance between two monomers in Hr octamer; native and MTSSLlabeled $\mathrm{Hr}$ are shown. The distance is measured between CYX50 $\mathrm{C} \alpha$ and Asp23 $\mathrm{C} \alpha$ Chains $\mathrm{A}$ and $\mathrm{B}$, respectively, in $\mathrm{Hr}$ octameric model. Black/Blue lines shows data and trend for $\mathrm{Hr}$ octamer model of reference. Brown/Red lines shows data and trend for Hr octamer model with MTSSL label.

The measured volume of the native octamer, at $110264 \AA^{3}$, was almost $3000 \AA^{3}$ smaller than of the MTSSL-labeled octamer (at $113082 \AA^{3}$ ) most of which can be assigned to the spin label itself. Likewise, there is an average $\sim 200 \AA^{2}$ increase in solvent accessible surface (SAS) per monomer in the MTSSL-labeled octamer compared to the native form, cf. Table 1.

Table 1. SAS analysis for the average post MD of octameric structures (in $\AA 2$ ).

\begin{tabular}{cccr} 
Structure & Chain & Native & MTSSL \\
\hline Monomer & A & 4714 & 5042 \\
Monomer & B & 4697 & 4829 \\
Monomer & C & 4677 & 4796 \\
Monomer & D & 4901 & 4776 \\
Monomer & E & 4791 & 4594 \\
Monomer & F & 4842 & 5096 \\
Monomer & G & 4722 & 4824 \\
Monomer & H & 4819 & 5251 \\
Average & & 4770 & 4901 \\
\hline
\end{tabular}


Solvent Accessible Surface area measurements for the iron, for its amino acid ligands, and for Cys51, are listed in Table 2. For the diiron site (ligands included), this is an important issue since the autoxidation rate of the iron-dioxygen complex in all oxygen -carrying proteins (Hr included)[16] is directly correlated with solvent exposure. This correlation is of course expected since liberation of molecular oxygen from the $\mathrm{Fe}(\mathrm{II})$-dioxygen complex implies a neutral hydrophobic departing ligand, while autoxidation implies the departure of a polar or anionic superoxide - hence favored by more hydrophilic/solvent-exposed environments.

In line with the data shown above, position 51 obviously increases its SAS when labeled. In the native form, its SAS is reduced to virtually zero, meaning that the residue is in a hydrophobic area - while the labeling leads to a notable solvent exposure $\left(40.7 \AA^{2}\right.$ cf.Table 2$)$, meaning that the presence of the spin label MTSSL creates space in between monomers leading to access of water.

Meanwhile, at the active site, the environment is seen to indeed change for some of the residues and only slightly for the iron. Indeed, the latter is as expected almost entirely non- exposed to solvent - a logical fact for a dioxygen-carrying protein as discussed above. Nevertheless, a slight increases in SAS of $\sim 3 \AA^{2}$ is still seen the MTSSL-labelled octamer versus the native version. Small changes are also seen in the solvent exposures of the amino acids serving as ligands to the iron; on average, the total increase is of $\sim 19 \AA^{2}$ - a very small value but nevertheless one that distinctly warns of the effects that chemical derivatization (or indeed other changes, such as site-directed mutagenesis) may have on the diiron site in $\mathrm{Hr}$ even when these changes occur relatively far from the first coordination sphere. In this respect the behavior of the respective iron-ligated amino acids does differ qualitatively: while His77 sees its SAS reduced by $10 \AA^{2}$ to zero when labeled in the octameric form, His54 and His73 see even more dramatic changes in the opposite directions - with the rest of the amino acids contributing much less. Perhaps of note is also the fact that these changes are not intrinsic to the monomer, but are rather the effect of the octameric environment. Indeed, the SAS of the iron ligands changes by very little in the opposite direction (increased hydrophobicity for MTSSL-Hr compared to native $\mathrm{Hr}$ ) when the MD simulation is performed on isolated monomers, as opposed to octamers. 
Table 2. SAS for selected residues inside averaged structures obtained from the sampling of $\mathrm{Hr}$ molecular dynamics monomers (mon) and octamers (oct) with MTSSL label (MTSSL) and without (native). Measurements are reported in $\AA^{2}$.

\begin{tabular}{ccccccccccccc} 
& & & & & His & His & Glu & His & His & His & Glu \\
Structure & $\mathrm{Fe} 1$ & $\mathrm{Fe} 2$ & $\mathrm{Fe}^{\mathrm{a}}$ & $\mathrm{Fe}^{\mathrm{a}}$ & 25 & 54 & 58 & 73 & 77 & 101 & 106 & $51^{\mathrm{b}}$ \\
\hline mon-nat & 0.0 & 0.6 & 0.0 & 0.6 & 2.8 & 1.1 & 0.0 & 9.3 & 9.7 & 7.6 & 0.0 & 11.3 \\
mon-MTSSL & 0.0 & 0.0 & 0.0 & 0.0 & 1.0 & 1.0 & 0.0 & 8.7 & 3.6 & 7.9 & 0.1 & 94.0 \\
oct-nat & 0.0 & 0.4 & 0.0 & 0.4 & 1.7 & 0.9 & 0.0 & 0.8 & 9.5 & 6.0 & 0.0 & 0.1 \\
oct-MTSSL & 0.0 & 1.1 & 0.1 & 2.2 & 1.4 & 10.2 & 0.3 & 14.2 & 0.0 & 10.9 & 0.5 & 40.7 \\
\hline
\end{tabular}

a Averaged measurement of SAS for the octamer structures.

${ }^{b}$ Position 51 in the protein, either Cys or Cys-MTSSL.

\section{CONCLUSIONS}

The first MM study of hemerythrin is reported here. The data provide support for interpreting experiments where $\mathrm{Hr}$ is a template or starting material for blood substitutes - hence, in multimeric form and chemically derivatized with polyethylene glycols, spin labels, or other reagents. Derivatization with a spin label at the native Cys51 position in $\mathrm{Hr}$, is found to destabilize the octameric structure and to lead to a slight increase in solvent accessibility of the diiron center. Both of these consequences of the chemical derivatization would be functional disadvantages for an oxygencarrying protein. This puts some limitations on the relevance of the EPR spin labeling studies with $\mathrm{Hr}$, and also suggests the need for a careful investigations of the effects of the PEGylation procedures in the context of blood substitute candidates.

\section{EXPERIMENTAL SECTION}

Classical molecular dynamics simulations were performed using YASARA.[17] Monomeric and octameric hemerythrin structures were modeled using the X-ray diffraction based structure PDB ID 114Y[18] from Phascolopsis gouldii wild type retrieved from the Protein Data Bank[19] website. In the case of the MTSSL-labeled Hr structures, the MTSSL model (cf. Figure 2) was created using GaussView version 5;[20] its geometry was energetically taken to a minimum at the semiempirical PM6 level of theory.[21] 


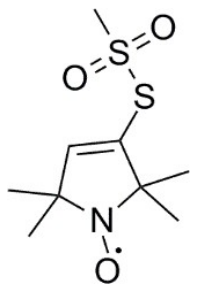

Figure 2. MTSSL, Full IUPAC nomenclature name:

(1-Oxyl-2,2,5,5-tetramethylpyrroline-3- methyl) methanethiosulfonate.

This model was then attached to each one of the monomers in Cys51 position of hemerythrin, yielding the structures illustrated in Figure 3 and Figure 4.
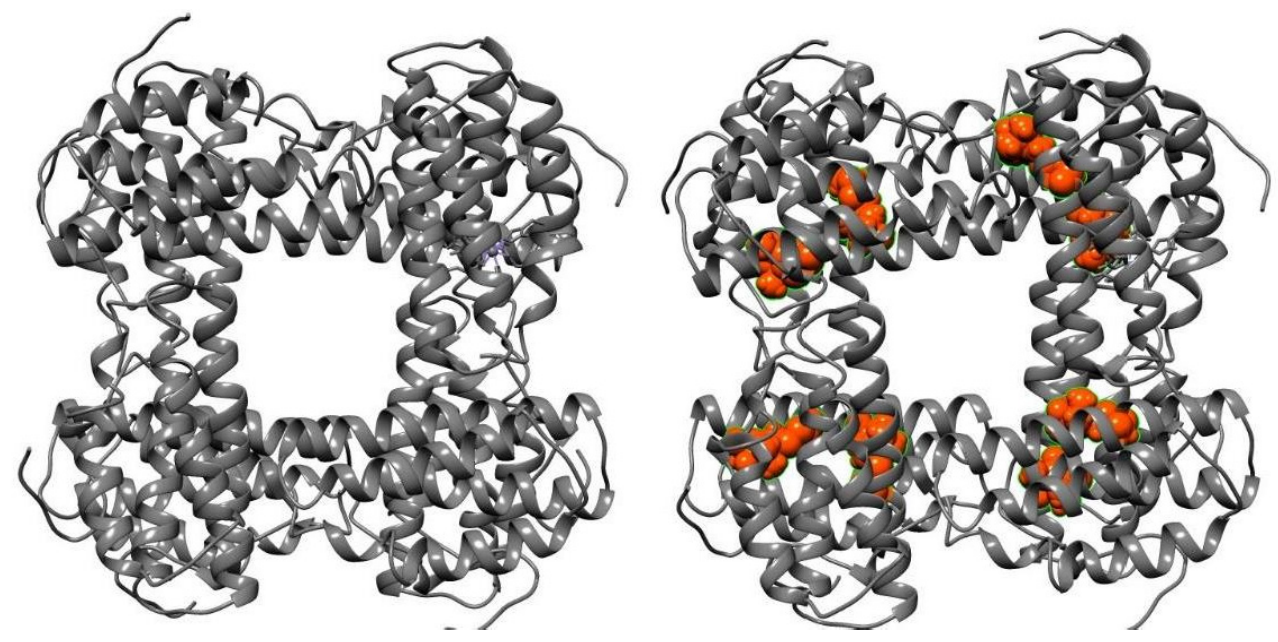

Figure 3. Left: Hr octamer, in ribbon representations. Right: Hr-octamer-MTSSL labelled at Cys51 position of each monomer. $\mathrm{Hr}$ represented in gray ribbons, MTSSL label in orange VDW spheres. All graphics have been constructed using UCSF Chimera software program.[22]

All simulations were carried out using the AMBER 03 force field as implemented in YASARA.[23] Long-range interactions were treated with the Particle Mesh Ewald[24] algorithm with a cut off value of $10.48 \AA$. Periodic boundary conditions were defined using a water filled box of $10 \AA$ distance from all the amino acids of the protein. Each system was solvated with TIP3P water molecules model.[25] Counter-ions of $\mathrm{NaCl}$ were randomly placed in the box to neutralize the system to physiological $\mathrm{pH}$ 7.4. Each system was subjected first to steepest descent minimization, this is 
hereafter referred to as the initial structure. After removing conformational stress through equilibration simulations, sampling was carried out with a time step of $0.5 \mathrm{fs}$ and with snapshots saved in the trajectory every $3 \mathrm{ps}$. All simulations were carried out at constant pressure and temperature (NPT) conditions at $298 \mathrm{~K}$, for a period of $1 \mathrm{~ns}$. After $10 \mathrm{~ns}$ MD simulations, were sampled. RMSD deviation for the trajectory was measured taking as reference the structure with minimum energy obtained during the trajectory, and for other calculations (for instance SAS and molecular volume) an averaged structure was obtained discarding the 5 nanoseconds of equilibration time and the first nanosecond of sampling.
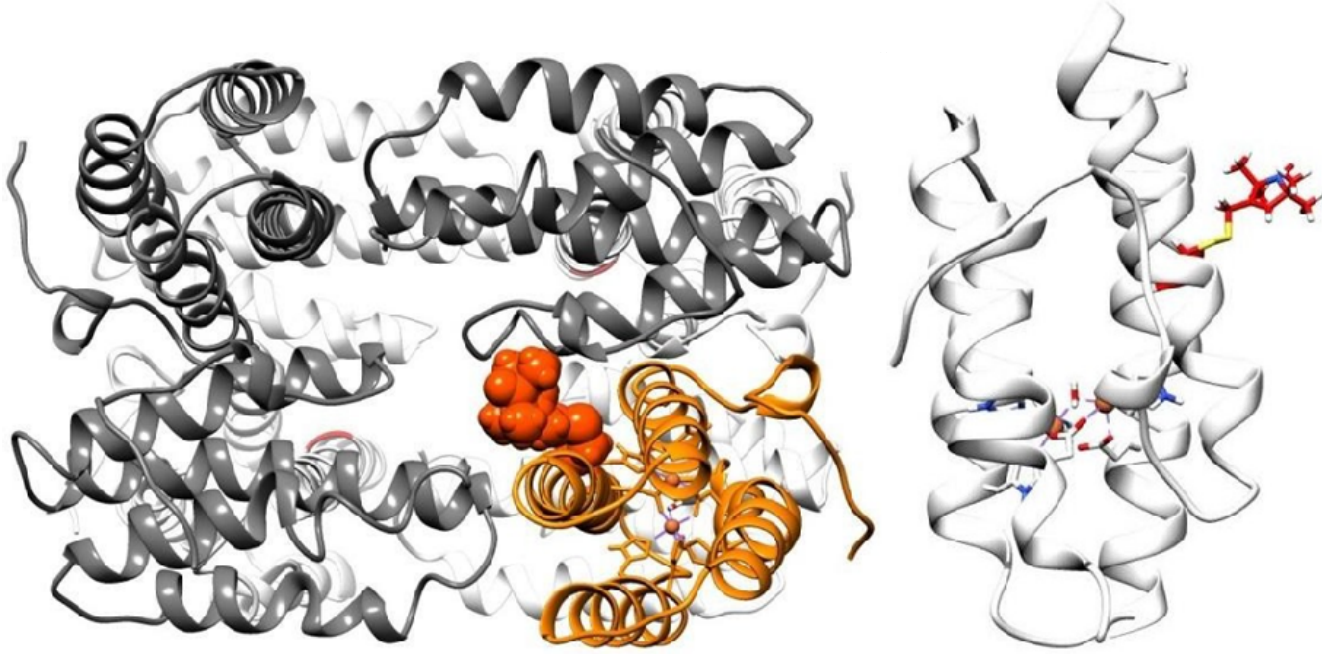

Figure 4. Left: Hr monomer-MTSSL (orange ribbons with MTSSL label in orange VDW surfaces) labeled at Cys51 position interacting with neighbor monomers (gray ribbons). Hr back monomers (not neighbors, not interacting with MTSSL label) are represented in withe ribbons for clarity. Right: Hr monomer (in white ribbons) MTSSL label in red ball and sticks, metallic center, $\mathrm{Fe}(\mathrm{Cl})-\mathrm{OH}-\mathrm{Fe}$, represented in balls and sticks.

\section{ACKNOWLEDGMENTS}

Funding from the Romanian Ministry of Education and Research (PN-IIIP2-2.1-PED2019-2293) is gratefully acknowledged. Infrastructure from the BabesBolyai University and from the European Centre for Bioinformatics and Genomics affiliated to Poznan University of Technology (Poznan, Poland) was used in this work. 


\section{REFERENCES}

1. D. Hathazi; A. C. Mot; A. Vaida; F. Scurtu; I. Lupan; E. Fischer-Fodor; G. Damian; D. M. Kurtz Jr.; R. Silaghi-Dumitescu; Biomacromolecules 2014, 15, 1920-1927.

2. S. V Kryatov; E. V Rybak-Akimova; S. Schindler; Chem Rev 2005, 105, 21752226.

3. E. Fischer-Fodor; A. Mot; F. Deac; M. Arkosi; R. Silaghi-Dumitrescu; J Biosci 2011, 36, 215-221.

4. M. Arkosi; F. Scurtu; A. Vulpoi; R. Silaghi-Dumitrescu; D. M. Kurtz Jr.; Artif Cells Blood Substitutes Biotechnol 2017, 45, 218-223.

5. A. C. Mot; A. Roman; I. Lupan; D. M. Kurtz Jr.; R. Silaghi-Dumitrescu; Protein J 2010, 29, 387-393.

6. V. A. Toma; A. D. Farcas; I. Roman; B. Sevastre; D. Hathazi; F. Scurtu; G. Damian; R. Silaghi-Dumitrescu; Int J Biol Macromol 2017, 107, 1422-1427.

7. F. Scurtu; B. Tebrean; M. K. Árkosi; A. Ionele; R. Silaghi-Dumitrescu; Stud Univ Babes-Bolyai Chem, 2019, 64(2), 421-434.

8. D. Hathazi; F. Scurtu; C. Bischin; A. Mot; A. Attia; J. Kongsted; R. SilaghiDumitrescu; Molecules 2018, 23, E350.

9. I. M. Takacs; A. Mot; R. Silaghi-Dumitrescu; G. Damian; J Mol Struct 2014, 1073, 18-23.

10. M. I. Takacs; A. Mot; R. Silaghi-Dumitrescu; G. Damian; Stud Univ BabesBolyai Chem 2013, 58, 61-69.

11. F. Pietra; Chem Biodivers 2017, 14, e1600158.

12. J. Dunne; A. Caron; P. Menu; A. I. Alayash; P. W. Buehler; M. T. Wilson; R. Silaghi-Dumitrescu; B. Faivre; C. E. Cooper; Biochem J 2006, 399, 513-524.

13. A. I. Alayash; Free Radic Res 2000, 33, 341-348.

14. A. I. Alayash; Trends Biotechnol. 2014, 32, 177-185.

15. D. A. Svistunenko; A. Manole; J Biomed Res 2020, 34, 281-291.

16. D. M. Kurtz; Essays Biochem 1999, 34, 85-100.

17.E. Krieger; G. Vriend; Bioinformatics 2014, 30, 2981-2982.

18. C. S. Farmer; D. M. Kurtz Jr.; Z. J. Liu; B. C. Wang; J. Rose; J. Ai; J. SandersLoehr; D. M. Kurtz Jr.; Z. J. Liu; B. C. Wang; J. Rose; J. Ai; J. Sanders-Loehr; J Biol Inorg Chem 2001, 6, 418-429.

19. H. M. Berman; J. Westbrook; Z. Feng; G. Gilliland; T. N. Bhat; H. Weissig; I. N. Shindyalov; P. E. Bourne; Nucleic Acids Res 2000, 28, 235-242.

20. R. Dennington; T. Keith; J. Millam; Semichem Inc., Shawnee Mission, KS 2009, Semichem Inc.

21. J. P. Stewart; J Mol Model 2007, 13, 1173-1213.

22. E. G. Pettersen; T. D. Goddard; C. C. Huang; G. S. Couch; D. M. Greenblatt; E. C. Meng; T. E. Ferrin; J Comput Chem 2004, 25, 1605-1612.

23. Y. Duan; C. Wu; S. Chowdhury; M. C. Lee; G. Xiong; W. Zhang; R. Yang; P. Cieplak; R. Luo; T. Lee; J. Caldwell; J. Wang; P. Kollman; J Comput Chem 2003, 24, 1999-2012.

24. U. Essmann; L. Perera; M. L. Berkowitz; T. Darden; H. Lee; L. G. Pedersen; J Chem Phys 1995, 103, 8577-8593.

25.W. L. Jorgensen; J Am Chem Soc 1981, 103, 335-340. 